\title{
Serum Gamma-Glutamyl Transferase Activity as a Potential Novel Cardiovascular Biomarker in COPD
}

\author{
Fulsen Bozkus MD, Nursel Dikmen MD, Hatice Sahin MD, and Anıl Samur PhD
}

\begin{abstract}
BACKGROUND: Gamma-glutamyl transferase (gamma-GT) is an enzyme present in the cell membranes, which is used as a new biomarker in prediction of inflammation, myocardial infarction, stroke, and cardiac death. The objective of this study was to investigate the relationship between serum levels of gamma-GT and cardiovascular disease in subjects with COPD and the correlation between serum gamma-GT level and degree of the limitation of air flow in COPD. METHODS: A total of 70 subjects $\mathbf{4 6 . 1 \%}$ ) with Global Initiative for Chronic Obstructive Lung Disease (GOLD) A-B and normal function of the liver and biliary tract (mean age [IQR] 59 [51.75-70] y; 77.1\% men) and 82 subjects (53.9\%) with GOLD C-D (mean age [IQR] 59 [56-66] y; 79.3\% men) participated. Serum levels of gamma-GT and C-reactive protein were measured and compared between the 2 groups. RESULTS: The serum level of gamma-GT was found to be significantly $(P<.001)$ higher in the GOLD stage $C$ and $D$ group than in the GOLD stage $A$ and $B$ group. Mean values of $C$-reactive protein, aspartate aminotransferase, and alanine aminotransferase did not differ significantly between the 2 groups. The prevalence of cardiovascular disease was statistically significantly higher in subjects in the GOLD stage $C$ and $D$ group than in the GOLD stage A and B group $(P<.001)$. The serum level of gamma-GT was higher in subjects with COPD with coexisting cardiovascular disease than in those without cardiovascular disease (64 units/L [interquartile range 57-72.5] vs 17.5 units/L [interquartile range 10-25]). CONCLUSIONS: Our results demonstrate that serum levels of gamma-GT may be helpful in grading the severity of COPD as the marker of oxidative stress, and there is a strong correlation between high serum levels of gamma-GT and cardiovascular events in subjects with COPD. Key words: gamma-glutamyl transferase; chronic obstructive pulmonary disease; cardiovascular disease. [Respir Care 2016;61(11):1465-1471. (C) 2016 Daedalus Enterprises]
\end{abstract}

\section{Introduction}

Today, COPD is recognized as the third leading cause of death worldwide. ${ }^{1}$ Patients with COPD are more likely to

\footnotetext{
Dr Bozkus is affiliated with the Department of Chest Diseases, Faculty of Medicine, Kahramanmaras Sutcu Imam University, Kahramanmaras, Turkey. Drs Dikmen and Sahin are affiliated with the Department of Chest Diseases, Kahramanmaras Necıp Fazıl State Hospital, Kahramanmaraş Merkez, Turkey. Dr Samur is affiliated with the Department of Biostatistics, Faculty of Medicine, Akdeniz University, Antalya, Turkey.

The authors have disclosed no conflicts of interest.
}

Correspondence: Fulsen Bozkus MD, Faculty of Medicine, Sutcu Imam University, Avsar Campus, 46100 Kahramanmaras, Turkey. E-mail: fulsenbatmaz@gmail.com.

DOI: $10.4187 /$ respcare. 04800 have preexisting cardiovascular disease and a higher risk of acute events, hospitalizations, and death from cardiovascular disease. ${ }^{2,3}$ Because of this strong relationship, the Global Initiative for Chronic Obstructive Lung Disease (GOLD) initiative recognizes cardiovascular disease as the most important coexisting disease with COPD and suggests that it should be routinely sought in patients with COPD but makes no recommendation regarding how this should be done. ${ }^{4}$

The discovery of new biomarkers that could be helpful in determination of cardiovascular risk in patients with COPD could help to develop personalized therapy for that specific phenotype. To be clinically practicable, these biomarkers should be easily measurable, noninvasive, and inexpensive. In this context, gamma-glutamyl transferase (gamma-GT) could be a promising biomarker to determine patients with COPD who have an increased risk for poor cardiovascular prognosis. ${ }^{5}$ 
Gamma-GT is an enzyme found in the cell membranes of numerous tissues, especially the liver, bile duct, and kidneys. Gamma-GT is well known to be increased in hepatobiliary dysfunction and in alcohol abuse. ${ }^{6}$ Previous studies have demonstrated that an increased level of gamma-GT is strongly associated with prognosis in cardiopulmonary disorders such as heart failure, acute myocardial infarction, and coronary artery disease. ${ }^{7,8}$

In light of all of this information, the objective of this study was to investigate the associations of serum gamma-GT level with degree of the limitation of air flow and cardiovascular events in subjects with COPD. We also examined the relationships between serum levels of gamma-GT and the pulmonary function test; Modified British Medical Research Council dyspnea scale (MMRC); history of exacerbation and hospitalization; and serum levels of C-reactive protein, alanine aminotransferase, and aspartate aminotransferase.

\section{Methods}

\section{Study Population and Study Design}

The study population consisted of subjects admitted to the Chest Diseases Clinic of Necip Fazıl State Hospital who were determined to have a confirmed diagnosis COPD based on the criteria established by the GOLD. According to the new recommendations by GOLD, $\mathrm{FEV}_{1}$ is not an adequate criterion alone in evaluation of disease severity. Accordingly, a combined evaluation system is recommended based on spirometric classification of subjects and/or risk of exacerbation in addition to the evaluation of symptoms. A COPD assessment test or the MMRC is recommended in the GOLD guidelines to evaluate symptoms. The GOLD criteria for COPD stages are defined as follows: (1) GOLD A: GOLD 1 or 2 (mild to moderate mild limitation of air flow) and/or $0-1$ exacerbation/y and no hospitalization due to exacerbation, MMRC $0-1$ or COPD assessment test (CAT) score <10; (2) GOLD B: GOLD 1 or 2 (mild to moderate mild limitation of air flow) and/or 0-1 exacerbation/y or no hospitalization due to exacerbation, MMRC $\geq 2$ or COPD assessment test score $\geq 10$; (3) GOLD C: GOLD 3 or 4 (severe or very severe limitation of air flow) and/or $\geq 2$ exacerbations/y or $\geq 1$ hospitalization due to exacerbation, MMRC $0-1$ or COPD assessment test score <10; (4) GOLD D: GOLD 3 or 4 (severe or very severe limitation of air flow) and/or $\geq 2$ exacerbations/y or $\geq 1$ hospitalization due to exacerbation, MMRC $\geq 2$ or COPD assessment test score $\geq 10 .{ }^{9}$ We divided the subjects with COPD into 2 groups: Group 1 consisted of the subjects with COPD with mild to moderate limitation of air flow (GOLD A-B), and Group 2 included the subjects with COPD with severe or very severe limitation of air flow (GOLD C-D).

\section{QUICK LOOK}

\section{Current knowledge}

COPD is a common and disabling disease and the third leading cause of global mortality after ischemic heart disease. Exacerbations of COPD accelerate pulmonary function decline, affecting the quality of life in patients with COPD, and, moreover, remain the major contributors to morbidity and mortality. Cardiovascular comorbidities are prevalent in patients with COPD and of great importance, since they have a negative impact on patients' health status.

\section{What this paper contributes to our knowledge}

There has been increasing interest in potential molecules that can be measured accurately and reproducibly to be used in COPD as biomarkers to predict clinically important outcomes. Such molecules have been successfully used in cardiovascular diseases; therefore, cardiac biomarkers have attracted attention for their potential use in COPD. The results of our study showed a strong association between high serum gamma-flutamyl transferase levels and cardiovascular events in subjects with COPD.

Patients with a history of hepatobiliary disorders, alcohol abuse, or any other acute diseases were excluded from the study. Demographics and medical history of subjects were collected utilizing a standardized questionnaire including metabolic and cardiovascular diseases, history of exacerbation and hospitalization in the last $1 \mathrm{y}$, and habits. Cardiovascular disease was identified in cases where the subjects had coronary artery disease, heart failure, or arrhythmia. Coronary artery disease was defined as having a clinical history of coronary artery disease, an abnormal outcome of stress test, including ischemia, or coronary stenosis $>50 \%$ identified on a coronary angiogram. Heart failure was defined as having a functionally or structurally disturbed ventricular filling or blood ejection. Arrhythmia was defined as having an irregular heart beat, including too fast or too slow heart rates.

Smoking history was assessed using pack-years and calculated by multiplying the number of daily consumed cigarettes by years of smoking. The study was approved by the ethics committee of Sutcu Imam University, and written informed consent was received from all subjects.

\section{Laboratory Analyses}

Blood samples were collected from the antecubital vein in each subject after overnight fasting. The gamma-GT 
Table 1. Demographic and Clinical Characteristics of the Study Group

\begin{tabular}{|c|c|c|c|}
\hline Characteristics & Group 1 GOLD A-B COPD & Group 2 GOLD C-D COPD & $P$ \\
\hline Age, median (IQR) y & $59(51.8-70)$ & $59(56-66)$ & .73 \\
\hline Male sex, $n(\%)$ & $54(77.1)$ & $65(79.3)$ & .86 \\
\hline CVD, $n(\%)$ & $32(45.7)$ & $64(78)$ & $<.001$ \\
\hline Smoking history, median (IQR) pack-years & $40(30-48)$ & $38(34.75-45)$ & .39 \\
\hline MMRC, median (IQR) & $1(1-2)$ & $2(1-3)$ & $<.001$ \\
\hline Exacerbation/hospitalization, median (IQR) & $0(0-1)$ & $1(0.75-2)$ & $<.001$ \\
\hline \multicolumn{4}{|l|}{ Traditional risk factors for CVD } \\
\hline Diabetes mellitus, $n(\%)$ & $10(14.3)$ & $11(13.4)$ & .01 \\
\hline Hypertension, $n(\%)$ & $25(35.7)$ & $55(67.1)$ & $<.001$ \\
\hline BMI, median (IQR) kg/m² & $24.75(22.5-27.45)$ & $24.65(22.5-28,18)$ & .98 \\
\hline \multicolumn{4}{|l|}{ Pulmonary function test results } \\
\hline FVC, median (IQR) L & $3.21(2.67-3.84)$ & $1.45(0.65-1.87)$ & $<.001$ \\
\hline FVC, median (IQR) \% predicted & $82.15(75-88.18)$ & $55(45-65)$ & $<.001$ \\
\hline $\mathrm{FEV}_{1}$, median (IQR) L & $2.35(2.06-2.62)$ & $0.88(0.4-1.32)$ & $<.001$ \\
\hline $\mathrm{FEV}_{1}$, median (IQR) \% predicted & $66.5(58-81)$ & $40(32-47.25)$ & $<.001$ \\
\hline $\mathrm{FEV}_{1} / \mathrm{FVC}$, median (IQR) & $64(58.8-66.8)$ & $57(52-65.5)$ & $<.001$ \\
\hline \multicolumn{4}{|l|}{ Laboratory variables } \\
\hline C-reactive protein level, median (IQR) $\mathrm{mg} / \mathrm{dL}$ & $9(5.38-15)$ & $10.5(6.75-15)$ & .39 \\
\hline Gamma-GT level, median (IQR) units/L & $28.5(15.5-58)$ & $63.5(48-74.25)$ & $<.001$ \\
\hline AST level, median (IQR)units/L & $21(14.75-31)$ & $18.5(13-27)$ & .24 \\
\hline ALT level, median (IQR) units/L & $23(14-31)$ & $20(12-28)$ & .30 \\
\hline $\begin{array}{l}\text { GOLD = Global Initiative for Chronic Obstructive Lung Disease } \\
\text { IQR }=\text { interquartile range } \\
\text { CVD = cardiovascular disease } \\
\text { MMRC = modified Medical Research Council dyspnea scale } \\
\text { BMI = body mass index } \\
\text { Gamma-GT = gamma-glutamyl transferase } \\
\text { AST }=\text { aspartate aminotransferase } \\
\text { ALT = alanine aminotransferase }\end{array}$ & & & \\
\hline
\end{tabular}

activity was measured with the Roche-Hitachi autoanalyzer using the original kits. In addition, the relationships of the pulmonary functions of these parameters were further studied.

\section{Statistical Analysis}

Statistical analysis was performed utilizing SPSS 18.0 (SPSS, Chicago, Illinois). The continuous variables were tested for normality of their distribution and expressed as median and interquartile ranges (IQRs), whereas the categorical variables are given as frequencies and percentages. The differences between 2 groups were compared using the Mann-Whitney test, whereas multiple groups were compared using one-way analysis of variance, followed by the Tukey post hoc test for multiple comparisons for subjects with COPD. The chi-square test was used to analyze categorical variables. Multivariate logistic regression analysis was performed to evaluate the risk factors for cardiovascular disease in COPD, incorporating all factors that showed values of $P<.05$ in the univariate analyses. The correlations between continuous variables were measured through Spearman correlation coefficients (r). All
$P$ values are 2 -sided, and $P$ values of $<.05$ were considered significant.

\section{Results}

A total of 152 subjects who met the selection criteria were enrolled in this study: $70(46.1 \%)$ with GOLD stages $\mathrm{A}$ and $\mathrm{B}$ and 82 (53.9\%) with GOLD stages C and D. The groups are described in Table 1. The groups showed no significant difference in terms of age, sex, body mass index, and total duration of smoking $(P=.73, P=.86$, $P=.98$, and $P=.39$, respectively). The mean $\mathrm{FEV}_{1}$ level of group 2 was $0.88(0.4-1.32) \mathrm{L}$, and the mean FVC level was found to be $1.45(0.65-1.87) \mathrm{L}$. The levels of $\mathrm{FEV}_{1}$ and FVC were found to be significantly lower in GOLD stage $\mathrm{C}$ and $\mathrm{D}$ group compared with the GOLD stage A and $\mathrm{B}$ group $(P<.001)$ (Table 1$)$.

The mean value of gamma-GT in COPD with coexisting group is 64 and the mean value of gamma-GT in Group 2 GOLD C and D COPD is 63.5. The level of gamma-GT was found to be significantly $(P<.001)$ higher in the GOLD stage $C$ and D group than in the GOLD stage A and B group. No statistically significant difference was 


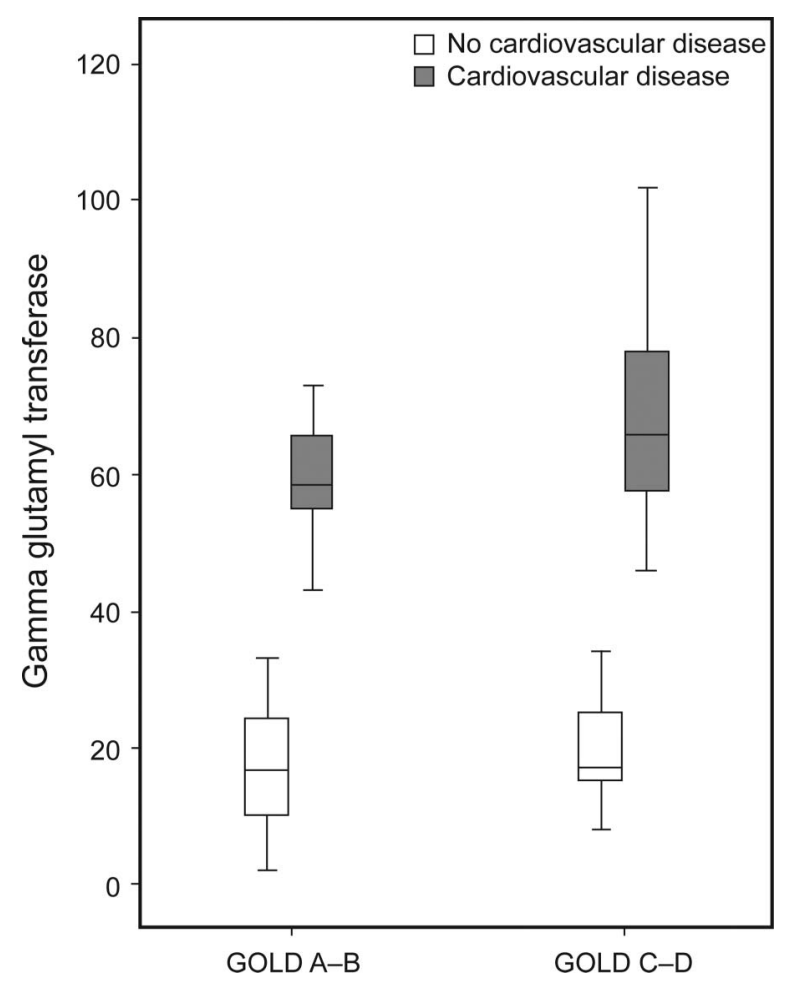

Fig. 1. Distribution of gamma-glutamyl transferase level in subjects with COPD with and without cardiovascular disease. Subjects were grouped as GOLD A-B and GOLD C-D. Center lines represent the median value, whereas the upper and lower border of each boxes represent the 3rd and 1st quartiles, respectively. Whiskers represent 5 th and 95th percentile.

found in the mean values of C-reactive protein, aspartate aminotransferase, and alanine aminotransferase between the 2 groups (Table 1). The prevalence of cardiovascular disease was statistically significantly higher in subjects in the GOLD stage $\mathrm{C}$ and $\mathrm{D}$ group compared with those in the GOLD stage A and B group $(P<.001)$ (Table 1$)$. Also, the prevalence of hypertension was found to be significantly higher in the GOLD stage $\mathrm{C}$ and $\mathrm{D}$ group $(P<.001)$ than in the GOLD stage A and B group. No statistically significant difference was found in the prevalence of diabetes mellitus between the 2 groups (Table 1). The serum level of gamma-GT was higher in subjects with COPD with coexisting cardiovascular disease than in those without cardiovascular disease (median 64 [IQR 57-72.5] units/L vs 17.5 [IQR 10-25] units/L) (Fig. 1). The optimal cutoff value of gamma-GT to have cardiovascular disease was measured as >42 IU/L, with $97 \%$ sensitivity and $98 \%$ specificity.

The level of gamma-GT was negatively correlated with $\mathrm{FEV}_{1}$ in subjects in the GOLD stage A and B group $(P=.004, \mathrm{r}=-0.343)$ (Fig. 2). There was a positive correlation between the level of gamma-GT and $\mathrm{FEV}_{1} / \mathrm{FVC}$ in subjects in GOLD stages $\mathrm{C}$ and $\mathrm{D}(P=.046$, $\mathrm{r}=-0.221$ ) (Fig. 3).

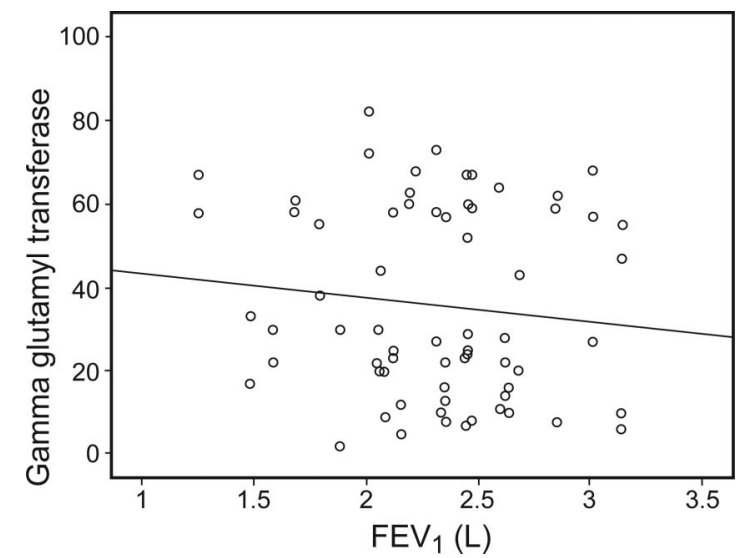

Fig. 2. Correlation of gamma-glutamyl transferase level and $\mathrm{FEV}_{1}$ in subjects with COPD with mild to moderate air flow limitation (GOLD A-B). $P<.001$.

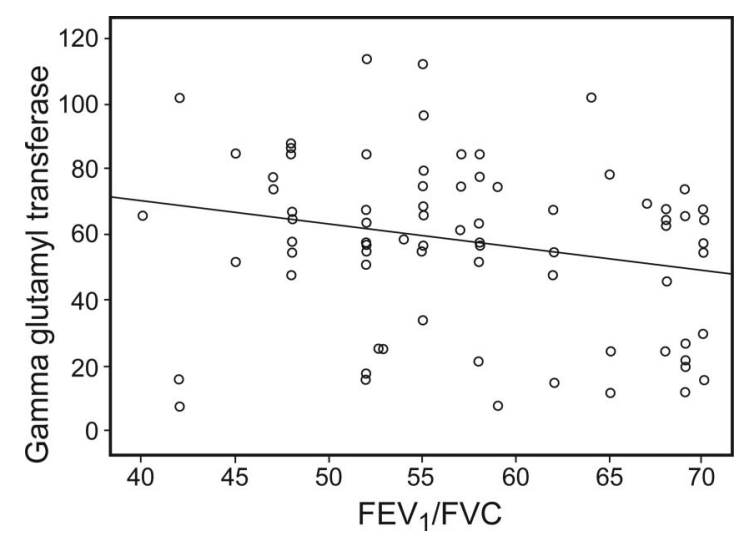

Fig. 3. Correlation of gamma-glutamyl transferase level and FEV in subjects with COPD with severe to very severe air flow limitation (GOLD C-D). $P<.001$.

The numbers for the MMRC and exacerbation/hospitalization were found to be significantly greater in the GOLD stage $\mathrm{C}$ and $\mathrm{D}$ group $(P<.001)$ compared with the GOLD stage A and B group. Gamma-GT was significantly different between MMRC groups in GOLD A-B $(P<.001)$. Pairwise comparison revealed that gamma-GT for MMRC 2 was significantly higher than in the MMRC $0(P=.001)$ and MMRC 1 groups $(P=.001)$. Also, gamma-GT was significantly different between MMRC groups in GOLD C-D $(P<.001)$. Pairwise comparison revealed that gamma-GT of MMRC $2(P=.002)$, MMRC $3(P<.001)$, and MMRC 4 were significantly higher than MMRC $1(P=.02)$.

The potential factors for identifying cardiovascular disease were further studied using an univariate procedure. Then all parameters found to be associated with cardiovascular disease with a significance level below 0.1 were evaluated in a multiple regression analysis. The final regression model included age, sex, hypertension, and diabetes mellitus, and in conclusion, independent predictors 


\section{Gamma-Glutamyl Transferase as a COPD Biomarker}

Table 2. Risk Factors for Cardiovascular Diseases in Subjects With COPD: Multivariate Analysis

\begin{tabular}{lccc}
\hline \hline \multicolumn{1}{c}{ Risk Factor } & Odds Ratio & $95 \%$ CI & $P$ \\
\hline Age $(\mathrm{y})$ & 1.00 & $0.96-1.05$ & .89 \\
Male sex & 0.32 & $0.1-0.99$ & .048 \\
C-reactive protein $(\mathrm{mg} / \mathrm{dL})$ & 1.00 & $0.94-1.08$ & .91 \\
Body mass index $\left(\mathrm{kg} / \mathrm{m}^{2}\right)$ & 0.86 & $0.75-0.99$ & .036 \\
Hypertension & 5.63 & $2.31-13.74$ & $<.001$ \\
Diabetes mellitus & 0.98 & $0.27-3.56$ & .98 \\
Exacerbation/hospitalization & 3.39 & $1.89-6.06$ & $<.001$ \\
\hline
\end{tabular}

of cardiovascular disease were found to be the number of exacerbations/hospitalizations and hypertension in subjects with COPD (Table 2).

\section{Discussion}

The present study yielded 2 major findings. First, the serum level of gamma-GT was found to be significantly higher in the GOLD stage $\mathrm{C}$ and $\mathrm{D}$ group compared with the GOLD stage A and B group. Second, subjects in the GOLD stage $\mathrm{C}$ and $\mathrm{D}$ group were found to show a higher prevalence of cardiovascular disease. To the best of our knowledge, this is the first study to compare the gamma-GT level by especially considering cardiovascular events and oxidative stress in subjects with COPD.

COPD is a chronic inflammatory lung disease that is known to have several systemic features, including an increased risk of cardiovascular disease. There is now considerable evidence regarding the relationship between COPD and cardiovascular disease. In a large cohort of subjects with COPD, the prevalence of coronary artery disease was significantly higher at $33.6 \%$ compared with a matched cohort without COPD, which had a $27.1 \%$ prevalence of coronary artery disease. ${ }^{10}$ Numerous population studies have demonstrated that the limitation of air flow as specified by $\mathrm{FEV}_{1}$ or $\mathrm{FEV}_{1} / \mathrm{FVC}$ is a predictor of cardiovascular risk. ${ }^{11} \mathrm{FEV}_{1}$ is also an independent predictor of cardiovascular mortality in COPD. The Lung Health Study ${ }^{2}$ reported that fatal coronary events were increased by $28 \%$ and nonfatal coronary events by $20 \%$ for every $10 \%$ decrease in $\mathrm{FEV}_{1}$ among subjects who had mild to moderate COPD. Similarly, in our study, the rate of cardiovascular disease was $78 \%$ in subjects with COPD with severe or very severe limitation of air flow, whereas this rate was found to be $45.7 \%$ in subjects with COPD who had mild to moderate limitation of air flow.

The exact mechanism of gamma-GT activity in cardiovascular system diseases is yet to be established. Among the most important mechanisms proposed for the relationship between gamma-GT and cardiovascular disease is the effects of gamma-GT on oxidative stress and glutathione mechanism. In one study, ${ }^{12}$ gamma-GT activity has been detected in the atheroma plaque of carotid and coronary arteries, and gamma-GT found in the atherosclerotic plaques has been suggested to play a role in the formation and rupture of the plaques via catalysis of the oxidation of LDL. In the Ludwigshafen Risk and Cardiovascular Health (LURIC) study, Stojakovic et al ${ }^{13}$ examined the association of gamma-GT with all-cause and cardiovascular mortality in 2,556 subjects with and 699 subjects without angiographic evidence of coronary artery disease. The authors demonstrated that serum levels of gamma-GT predict total and cardiovascular mortality in persons with coronary artery disease independently of other cardiovascular risk factors.

The exact mechanism responsible for the increased risk of cardiovascular disease in patients with COPD is not clear; however, numerous mechanisms have been proposed, including systemic inflammation. It is well known that COPD is associated not only with pulmonary but also with systemic inflammation. It has been proposed that systemic inflammation may also play a role in the extrapulmonary features associated with COPD, such as increased risk of cardiovascular disease. ${ }^{14}$ When increased systemic inflammation is present, patients with COPD are particularly susceptible to vascular events following an exacerbation. ${ }^{15}$ Thus, cardiovascular risk associated with COPD may be reduced along with a decrease in the frequency of exacerbation. The serum level of C-reactive protein is positively correlated with the level of gamma-GT, suggesting that there may be an underlying association between oxidative stress and general inflammation-exacerbated COPD. ${ }^{16}$ A strong correlation between C-reactive protein and gamma-GT was described for the first time by Lee et al. ${ }^{17}$ In their study on a large healthy population, the authors found a correlation between C-reactive protein and increased gamma-GT, which is one of the markers of oxidative stress. Numerous subsequent studies investigated the association between C-reactive protein and gamma-GT and reported a positive correlation. ${ }^{16,17}$ Additionally, in a study by Ulus et al ${ }^{18}$ conducted on subjects with acute coronary syndrome, high serum level of gamma-GT level was found to be an independent predictor for major cardiovascular events over 1- and 6-month follow-up periods and a better marker than C-reactive protein, whereas a positive correlation was reported between serum levels of C-reactive protein and gamma-GT. In our study, the level of gamma-GT was found to be much higher in the GOLD stage $\mathrm{C}$ and D group than in the GOLD stage A and B group. In addition, serum level of gamma-GT was higher in subjects with COPD with cardiovascular disease compared with those without cardiovascular disease (64 units/L [IQR 57-72.5] units/L vs 17.5 units/L [IQR 10-25] units/L). However, we did not find any significant correlation between C-reactive protein and cardiovascular dis- 


\section{Gamma-Glutamyl Transferase as a COPD Biomarker}

ease in subjects with COPD, which might be due to sample size, subject selection, and inclusion-exclusion criteria of the study.

Oxidative stress is another mechanism responsible for an increased risk of cardiovascular disease in subjects with COPD. COPD has been associated with both local pulmonary and systemic oxidative stress. ${ }^{19}$ Ischemic heart disease has also been associated with systemic oxidative stress. Several traditional risk factors, such as hypertension, diabetes, hypercholesterolemia, and smoking, are associated with increased production of oxygen free radicals from the smooth muscle cells and vascular endothelium. Reactive oxygen species have been shown to cause atherosclerosis through numerous mechanisms. ${ }^{14}$

In systemic inflammation, the circulating oxidants increase, whereas the antioxidant capacity decreases. Antioxidants are found in the systemic circulation (ascorbate and glutathione) and the epithelial lining fluid (glutathione, ceruloplasmin, ascorbic acid, and mucin). Xenobiotic-metabolizing enzymes support the body to protect against oxidative stress. Glutathione $S$-transferase is among these enzymes. Gamma-GT, which has an important role in antioxidant defense, is an enzyme responsible for the extracellular catabolism of glutathione, and the level of gamma-GT is a commonly used diagnostic test for liver diseases in clinical practice. ${ }^{20}$ In general, an abnormally elevated gamma-GT level is considered as a marker of alcohol abuse and liver damage. However, these factors cannot explain the relationships of gamma-GT with pulmonary function and COPD, because $\mathrm{FEV}_{1}$ and FVC are clearly decreased, and the prevalence of COPD increased the subjects who had normal alanine aminotransferase levels and irregular alcohol consumption habits. ${ }^{21}$ The mechanism or mechanisms that link serum gamma-GT, which is a type of liver enzyme from a conventional viewpoint, to pulmonary function and COPD are yet to be clarified. One possible explanation is that the serum level of gamma-GT is associated with the pulmonary function as an early and sensitive biomarker of oxidative stress in humans. ${ }^{22}$ Also, chronic systemic oxidative stress is the primary pathophysiological mechanism for COPD. Because of its role in the degradation of antioxidant glutathione, gamma-GT is recognized as a biomarker of oxidative stress. ${ }^{23}$ In the present study, the serum level of gamma-GT was significantly higher in subjects with COPD with severe and very severe limitation of air flow than in those with mild to moderate limitation of air flow. In addition, there was a negative correlation between $\mathrm{FEV}_{1}$ and gamma-GT level of subjects with COPD with mild to moderate limitation of air flow and between $\mathrm{FEV}_{1} / \mathrm{FVC}$ of subjects with COPD with severe to very severe limitation of air flow.

Among the traditional risk factors of the development of cardiovascular disease, smoking is the causative factor in the majority of individuals with COPD and is an effector in the development of coronary artery disease. Because COPD and smoking are inextricably linked, it is difficult to show that the increased risk of cardiovascular disease is due to COPD alone in subjects with COPD. It is also very difficult to correct for cigarette smoke exposure statistically in studies. So, smoking seems likely to play an important role in the development of cardiovascular disease in COPD. ${ }^{14}$ In our study, there was no significant difference between the groups in terms of total duration of smoking.

Other traditional cardiovascular risk factors are also common in COPD. In the Atherosclerosis Risk in Communities Study, investigators have reported an increased prevalence of hypertension and diabetes in subjects with COPD compared with healthy individuals, which was even more evident in GOLD stages C and D. ${ }^{24}$ Consistently, in our study, also the prevalence of hypertension was higher in GOLD stages C and D, but no significant difference was found between the groups in terms of diabetes mellitus.

There are some limitations of our study. This is a crosssectional study, and the potential causal relationship between COPD and high serum gamma-GT levels cannot be concluded. However, the findings of this study have some important implications for the identification of cardiovascular outcomes in COPD stages and for the management and prognosis of these patients.

The results of our study indicate that gamma-GT may be helpful in grading the severity of COPD. The related increase in this marker may represent a possible pathophysiological mechanism underlying the increased cardiovascular morbidity of patients with COPD and detecting earlier the cases at a higher risk for developing the associated cardiovascular complications. Further prospective studies with a greater number of subjects are warranted to better clarify this issue.

\section{REFERENCES}

1. Lozano R, Naghavi M, Foreman K, Lim S, Shibuya K, Aboyans V, et al. Global and regional mortality from 235 causes of death for 20 age groups in 1990 and 2010: a systematic analysis for the Global Burden of Disease Study 2010. Lancet 2012;380(9859):2095-2128.

2. Anthonisen NR, Connett JE, Enright PL, Manfreda J, Lung Health Study Research Group. Hospitalizations and mortality in the Lung Health Study. Am J Respir Crit Care Med 2002;166(3):333-339.

3. Calverley PM, Anderson JA, Celli B, Ferguson GT, Jenkins C, Jones $\mathrm{PW}$, et al. Salmeterol and fluticasone propionate and survival in chronic obstructive pulmonary disease. N Engl J Med 2007;356(8): 775-789.

4. Vestbo J, Hurd SS, Agustí AG, Jones PW, Vogelmeier C, Anzueto A, et al. Global strategy for the diagnosis, management and prevention of chronic obstructive pulmonary disease: NHLBI/WHO Global Initiative for chronic obstructive pulmonary disease (GOLD): GOLD Executive Summary. Am J Respir Crit Care Med 2013;187(4):347365 .

5. Fraser A, Harris R, Sattar N, Ebrahim S, Smith GD, Lawlor DA. Gammaglutamyltransferase is associated with incident vascular 


\section{Gamma-Glutamyl Transferase as a COPD Biomarker}

events independently of alcohol intake analysis of the british women's heart and health study and meta-analysis. Arterioscler Thromb Vasc Biol 2007;27(12):2729-2735.

6. Whitfield JB. Gamma glutamyl transferase. Crit Rev Clin Lab Sci 2001;38(4):263-355.

7. Emdin M, Passino C, Michelassi C, Titta F, L'abbate A, Donato L, et al. Prognostic value of serum gamma-glutamyl transferase activity after myocardial infarction. Eur Heart J 2001;22(19):1802-1807.

8. Poelzl G, Eberl C, Achrainer H, Doerler J, Pachinger O, Frick M, Ulmer H. Prevalence and prognostic significance of elevated gamma-glutamyltransferase in chronic heart failure. Circ Heart Fail 2009;2(4):294-302.

9. Global Strategy for the Diagnosis, Management and Prevention of COPD, Global Initiative for Chronic Obstructive Lung Disease (GOLD) 2011. http://www. goldcopd.org/.

10. Mapel DW, Dedrick D, Davis K. Trends and cardiovascular comorbidities of COPD patients in the Veterans Administration Medical System, 1991-1999. COPD 2005;2(1):35-41.

11. Sin DD, Man SF. Chronic obstructive pulmonary disease as a risk factor for cardiovascular morbidity and mortality. Proc Am Thorac Soc 2005;2(1):8-11.

12. Emdin M, Pompella A, Paolicchi A. Gamma-glutamyltransferase, atherosclerosis and cardiovascular disease: triggering oxidative stress within the plaque. Circulation 2005;112(14):2078-2080.

13. Stojakovic T, Scharnagl H, Trauner M, Pieske B, Wellnitz B, Seelhorst $\mathrm{U}$, et al. Serum gammaglutamyl transferase and mortality in persons undergoing coronary angiography: the Ludwigshafen Risk and Cardiovascular Health Study. Atherosclerosis 2010;208(2):564571

14. Maclay JD, MacNee W. Cardiovascular Disease in COPD. Chest 2013;143(3):798-807.
15. Hurst JR, Hagan G, Wedzicha JA. Mechanism of statin associated mortality reduction in COPD. Chest 2007;132(4):1409-1410.

16. Holme J, Dawkins PA, Stockley EK, Parr DG, Stockley RA. Studies of gamma-glutamyl transferase in alpha-1 antitrypsin deficiency. COPD 2010;7(2):126-132.

17. Lee DH, Jacobs DR Jr. Association between serum gamma-glutamyltransferase and C-reactive protein. Atherosclerosis 2005;178(2): 327-330.

18. Ulus T, Yildirir A, Sade LE, Temiz A, Polat E, Bozbaş H, et al. Serum gamma-glutamyl transferase activity: new high-risk criteria in acute coronary syndrome patients? Coron Artery Dis 2008;19(7): 489-495.

19. MacNee W. Pulmonary and systemic oxidant/antioxidant imbalance in chronic obstructive pulmonary disease. Proc Am Thorac Soc 2005; 2(1):50-60.

20. Ermis H, Celik MR, Gulbas G, Tavli D, Aytemur ZA. Relationship between serum $\gamma$-glutamyltransferase levels and acute exacerbation of chronic obstructive pulmonary disease Pol Arch Med Wewn 2013; 123(3):85-90.

21. Kim HW, Lee SH, Lee DH. Relationship of serum gamma-glutamyltransferase levels with pulmonary function and chronic obstructive pulmonary disease. Lung 2014;192(5):719-727.

22. Lee DH, Blomhoff R, Jacobs DR Jr. Is serum gamma glutamyltransferase a marker of oxidative stress?. Free Radic Res 2004;38(6):535539

23. Paolicchi A, Franzini M, Emdin M, Passino C, Pompella A. The potential roles of gamma-glutamyltransferase activity in the progression of atherosclerosis and cardiovascular diseases. Vasc Dis Prev 2006;3(3):205-209.

24. Mannino DM, Thorn D, Swensen A, Holguin F. Prevalence and outcomes of diabetes, hypertension and cardiovascular disease in COPD. Eur Respir J 2008;32(4):962-969.

This article is approved for Continuing Respiratory Care Education credit. For information and to obtain your CRCE

(free to AARC members) visit

www.rcjournal.com

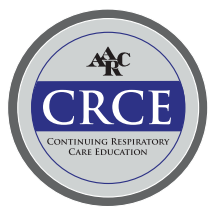

